Pacific Journal of Mathematics

LE PROBLÈME DE LÉVI POUR LES FIBRÉS
GRASSMANNIENS ET LES VARIÉTÉS DRAPEAU

K. ADACHI 


\title{
LE PROBLÈME DE LÉVI \\ POUR LES FIBRÉS GRASSMANNIENS \\ ET LES VARIÉTÉS DRAPEAUX
}

\author{
KENZō ADACHI
}

\begin{abstract}
In this paper we study the Levi problem for domains over the bundle whose fiber is a Grassmann manifold and whose base is a Stein manifold, and for domains over the flag manifold.
\end{abstract}

Introduction. Oka [9] a résolu le problème de Lévi pour des domaines au-dessus de $C^{n}$. Docquier-Grauert [3] ont résolu ce problème pour des domaines au-dessus d'une variété de Stein. Hirschowitz [5], [6], [7] a étudié le problème de Lévi pour des domaines au-dessus d'une variété infinitésimalement homogène. En particulier, Hirschowitz a démontré que tout ouvert non compact localement pseudoconvexe d'une variété grassmannienne est de Stein. Ueda [10] a démontré qu'un domaine localement pseudoconvexe au-dessus d'une variété grassmannienne $X$ qui n'est pas appliqué homéomorphiquement sur $X$ est une variété de Stein. D'autre part, Brun [1] a étudié le problème de Lévi pour des ouverts d'un fibré analytique localement trivial à base une variété de Stein et à fibre une variété homogène compacte. Dans ce papier, on étudié le problème de Lévi pour des domaines au-dessus d'un fibré à base une variété de Stein et à fibre une variété grassmannienne, et pour des domaines au-dessus d'une variété drapeau.

1.

Definition 1. Soient $X$ une variété complexe, $E$ un espace Hausdorff connexe, et $\Phi$ une application localement homéomorphe de $E$ dans $X$. Alors on dit que $\varepsilon=(E, \Phi, X)$ est un domaine étalé au-dessus de $X$, ou simplement un domaine.

Pour la définition du point frontière d'un domaine, nous référons à Grauert-Remmert [4] (Definition 4).

Definition 2. On dit qu'un domaine $\varepsilon=(E, \Phi, X)$ est localement pseudoconvexe si pour chaque point frontière $q$ il existe un voisinage $U$ de $q$ tel que $U \cap E$ soit une variété de Stein. 
Soit $\varepsilon=(E, \Phi, X)$ un domaine. On désigne l'ensemble des points frontières de $\varepsilon$ par $\partial E$. Alors on peut définir une structure de l'espace Hausdorff dans $\tilde{E}=E \cup \partial E$ et l'application continue $\tilde{\Phi}$ de $\tilde{E}$ dans $X$ telles que $\tilde{\Phi} \mid E=\Phi$.

Soient $S$ un ensemble analytique de positive codimension dans une variété complexe $X$ et $\varepsilon=(E, \Phi, X)$ un domaine. On dit qu'un point frontière $q$ est remuable le long de $S$ s'il existe un voisinage $U$ de $q$ tel que $(U, \tilde{\Phi} \mid U, X)$ soit un domaine univalent et que $\partial E \cap U$ soit contenu dans $\tilde{\Phi}^{-1}(S)$. Alors on obtient le lemme suivant.

LEMME 1. Soit $S$ un sous-ensemble analytique complexe de positive codimension dans une variété $X$. Soient $\varepsilon=(E, \Phi, X)$ un domaine et $q \in \partial E$ un point frontière remuable le long de $S$. Alors il existe un voisinage $V$ de $\Phi(q)$ et une section $\sigma$ de $\varepsilon$ sur $V-S$.

On désigne par $R$ l'ensemble des points frontières remuables le long de $S$. On pose $E^{*}=E \cup R$ et $\Phi^{*}=\tilde{\Phi} \mid E^{*}$. On dit qu'un domaine $\varepsilon^{*}=\left(E^{*}, \Phi^{*}, X\right)$ est l'extension de $\varepsilon$.

On a la proposition suivante due à Grauert-Remmert [4].

Proposition 1. Soient $S$ un ensemble analytique complexe de positive codimension dans une variété $X$ et $\varepsilon=(E, \Phi, X)$ un domaine. Supposons que $\varepsilon$ soit localement pseudoconvexe à tous les points au-dessus de $X-S$. Alors

(1) $S$ 'il n'existe pas de point frontière remuable le long de $S$, alors $\varepsilon$ est un domaine localement pseudoconvexe.

(2) Soit $\varepsilon^{*}=\left(E^{*}, \Phi^{*}, X\right)$ l'extension de $\varepsilon$ le long de $S$. Alors $\varepsilon^{*}$ est un domaine localement pseudoconvexe.

De plus (2) $\Rightarrow(1)$.

2. Soit $V_{n, r}(n \geq r)$ l'ensemble des $(n, r)$-matrices dont les rangs sont $\mathrm{r}$. Soit $G_{n, r}$ la variété grassmannienne. On va démontrer le théorème suivant.

TheOREME 1. Soit $(X, S, \pi)$ un fibré localement trivial à fibre une variété grassmannienne $G_{n, r}$ et à base une variété de Stein $S$. Soit $(D, \Phi, X)$ un domaine localement pseudoconvexe. $S$ 'il n'existe aucun ouvert $U$ de $D$ tel que $U$ soit appliqué homéomorphiquement sur un ouvert $\pi^{-1}(W), W$ un ouvert de $S$, alors D est de Stein. 
Démonstration. D'abord on a que iso $\left(G_{n, r}\right)=\operatorname{PGL}(n)$ si $2 r \neq n$ et $\operatorname{iso}\left(G_{n, r}\right) / \operatorname{PGL}(n)=Z / 2 Z$ si $2 r=n$ (voir par exemple J. Dieudonne [2]). Soit $\tilde{S}=\left\{(s, g): s \in S, g \in \operatorname{iso}\left(G_{n, r}, \pi^{-1}(s)\right)\right\}$. Soit $\nu_{1}: \tilde{S} \rightarrow S$ la projection canonique. Si $2 r \neq n,\left(\tilde{S}, S, \nu_{1}\right)$ est un fibré principal holomorphe à groupe structural $\operatorname{PGL}(n)$. Si $2 r=n$, la factorisation de Stein de $\tilde{S} \rightarrow S$ s'écrit $\tilde{S} \stackrel{u}{\rightarrow} \bar{S} \stackrel{v}{\rightarrow} S$ où $v$ est un revêtement non ramifié à deux feuillets et $u$ est un fibré principal à groupe structural $\operatorname{PGL}(n)$. Donc pour que $D$ soit de Stein, il faut et il suffit que $v^{*} D$ soit de Stein. Donc sans perte de généralité, on peut supposer que le groupe structural est $\operatorname{PGL}(n)$. On pose $\tilde{X}=\left\{((s, g), x):(s, g) \in \tilde{S}, x \in \pi^{-1}(s)\right\}$. Alors on obtient le diagramme commutatif:

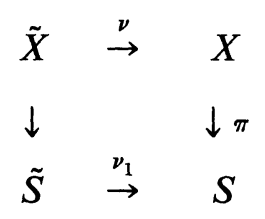

et $(\tilde{X}, X, \nu)$ est un espace fibré principal holomorphe à groupe structural $\operatorname{PGL}(n)$. Soit $\mathrm{U}(n)$ le groupe unitaire. Soient $\mathrm{gl}(n)$ et $\mathrm{u}(n)$ les algèbres de Lie de $\mathrm{GL}(n, C)$ et de $\mathrm{U}(n)$, respectivement. Alors

$$
\mathrm{gl}(n) / C=\mathrm{u}(n) / \sqrt{-1} R+\sqrt{-1} \mathrm{u}(n) / \sqrt{-1} R
$$

et

$$
\mathrm{u}(n) / \sqrt{-1} R \cap \sqrt{-1}(\mathrm{u}(n) / \sqrt{-1} R)=(0) .
$$

Donc $\operatorname{PGL}(n)$ est le complexifié d'un sous-groupe compact maximal $\mathrm{u}(n) / \mathrm{u}(1)$ de $\operatorname{PGL}(n)$. En particulier, $\operatorname{PGL}(n)$ est une variété de Stein. On définit une application $\theta: \tilde{S} \times G_{n, r} \rightarrow \tilde{X} \operatorname{par} \theta((s, g), t)=((s, g), g(t))$. Alors $\theta$ est l'application biholomorphe de $\tilde{S} \times G_{n, r}$ sur $\tilde{X}$. Soit $\tau_{1}: V_{n, r} \rightarrow$ $G_{n, r}$ l'application canonique. On définit une application $\tau: \tilde{S} \times V_{n, r} \rightarrow$ $\tilde{S} \times G_{n, r} \operatorname{par} \tau(x, y)=\left(x, \tau_{1}(y)\right)$. On pose

$$
\begin{gathered}
D_{1}=\tilde{X} \times{ }_{X} D, \quad D_{2}=\left(\tilde{S} \times G_{n, r}\right) \times_{\tilde{X}} D_{1}, \\
D_{3}=\left(\tilde{S} \times V_{n, r}\right) \times_{\left(\tilde{S} \times G_{n, r}\right)} D_{2} .
\end{gathered}
$$

Alors on obtient le diagramme commutatif:

$$
\begin{array}{ccccccc}
D_{3} & \stackrel{\tilde{\tau}}{\rightarrow} & D_{2} & \stackrel{\tilde{\rightarrow}}{\rightarrow} & D_{1} & \stackrel{\tilde{\nu}}{\rightarrow} & D \\
\downarrow \Phi_{3} & & \downarrow \Phi_{2} & & \downarrow \Phi_{1} & & \downarrow \Phi \\
\tilde{S} \times V_{n, r} & \stackrel{\tau}{\rightarrow} & \tilde{S} \times G_{n, r} & \stackrel{\theta}{\rightarrow} & \tilde{X} & \stackrel{\nu}{\rightarrow} & X
\end{array}
$$

Alors $\left(D_{3}, \Phi_{3}, \tilde{S} \times V_{n, r}\right)$ est un domaine localement pseudoconvexe. D'après le raisonnement de Ueda [10], $\left(D_{3}, \Phi_{3}, \tilde{S} \times C^{n r}\right)$ est un domaine 
localement pseudoconvexe. Grâce au théorème de Docquier-Grauert [3], $D_{3}$ est de Stein. Grâce au théorème de Matsushima-Morimoto [8], $D$ est de Stein. Ceci achève la démonstration du théorème 1.

3. Soit

$$
F=\left\{\left(g_{1}, \ldots, g_{p}\right) \in G_{n, r_{1}} \times G_{n, r_{2}} \times \cdots \times G_{n, r_{p}}: g_{1} \subset g_{2} \subset \cdots \subset g_{p}\right\}
$$

$\left(0<r_{1}<\cdots<r_{p}<n\right)$ une variété drapeau. Soient

$$
p_{i}: F \rightarrow G_{n, r_{1}} \times \cdots \times G_{n, r_{i-1}} \times G_{n, r_{i+1}} \times \cdots \times G_{n, r_{p}}
$$

les projections canoniques. Alors on obtient le théorème suivant:

TheOREME 2. Soit $(D, \Phi, F)$ un domaine localement pseudoconvexe. Alors $D$ est de Stein ou pour quelque $i, 1 \leq i \leq p$, il existe une section de $\Phi$ au-dessus de $p_{i}^{-1}\left(U_{i}\right)$, où $U_{i}$ est un ouvert de

$$
G_{n, r_{1}} \times \cdots \times G_{n, r_{i-1}} \times G_{n, r_{i+1}} \times \cdots \times G_{n, r_{p}} .
$$

Démonstration. Supposons qu'il n'existe aucune section de $p_{i}^{-1}\left(U_{l}\right)$ pour tout ouvert $U_{i}$ de

$$
G_{n, r_{1}} \times \cdots \times G_{n, r_{i-1}} \times G_{n, r_{i+1}} \times \cdots \times G_{n, r_{p}}
$$

et tout $i, 1 \leq i \leq p$. Soit $s \in V_{n, k}$. Alors on peut considérer la matrice $s$ comme une application de $C^{k}$ dans $C^{n}$ et l'image $\operatorname{Im} s$ comme un sous-espace vectoriel complexe de dimension $k$ dans $C^{n}$. On définit une application

$$
\pi: V_{n, r_{p}} \times V_{r_{p}, r_{p-1}} \times \cdots \times V_{r_{2}, r_{1}} \rightarrow F
$$

par

$$
\pi\left(t_{p}, t_{p-1}, \ldots, t_{1}\right)=\left(\operatorname{Im}\left(t_{p} t_{p-1} \cdots t_{1}\right), \ldots, \operatorname{Im}\left(t_{p} t_{p-1}\right), \operatorname{Im} t_{p}\right) .
$$

Alors $\left(V_{n, r_{p}} \times V_{r_{p}, r_{p-1}} \times \cdots \times V_{r_{2}, r_{1}}, F, \pi\right)$ est un espace fibré principal holomorphe à groupe structural GL( $\left.r_{p}\right) \times \mathrm{GL}\left(r_{p-1}\right) \times \cdots \times \mathrm{GL}\left(r_{1}\right)$. Soit $\tilde{D}$ un produit fibré de $V_{n, r_{p}} \times \cdots \times V_{r_{2}, r_{1}}$ et $D$ sur $F$. Alors on obtient le diagramme commutatif:

$$
\begin{array}{ccc}
\tilde{D} & \stackrel{\tilde{\pi}}{\rightarrow} & D \\
\downarrow \tilde{\Phi} & & \downarrow \Phi \\
V_{n, r_{p}} \times \cdots \times V_{r_{2}, r_{1}} & \stackrel{\pi}{\rightarrow} & F
\end{array}
$$


Alors $\left(\tilde{D}, \tilde{\Phi}, V_{n, r_{p}} \times \cdots \times V_{r_{2}, r_{2}}\right)$ est un domaine localement pseudoconvexe. On va démontrer que $\left(\tilde{D}, \tilde{\Phi}, C^{n r_{p}} \times V_{r_{p}, r_{p-1}} \times \cdots \times V_{r_{2}, r_{1}}\right)$ est un domaine localement pseudoconvexe. Soit

$$
T=\left(C^{n r_{p}}-V_{n, r_{p}}\right) \times V_{r_{p}, r_{p-1}} \times \cdots \times V_{r_{2}, r_{1}} .
$$

Soit $R$ l'ensemble des points frontières remuables le long de $T$. Supposons que $R$ ne soit pas vide. D'après la méthode de Ueda, il existe un point $q_{0} \in R$, un voisinage $U$ de $q_{0}$, et un voisinage $V$ de $\left(0, t_{0}\right)$ où $0 \in C^{n r_{p}}$, $t_{0} \in V_{r_{p}, r_{p-1}} \times \cdots \times V_{r_{2}, r_{1}}$ tels que $U$ soit appliqué homéomorphiquement sur $V$ par $\tilde{\Phi}$. Il existe un ouvert $B$ de $G_{n, r_{1}} \times \cdots \times G_{n, r_{p-1}}$ tel que

$$
\pi \tilde{\Phi}(U \cap \tilde{D})=\left\{\left(g_{1}, \ldots, g_{p}\right) \in B \times G_{n, r_{p}}: g_{1} \subset \cdots \subset g_{p}\right\} .
$$

Alors

$$
\pi \tilde{\Phi}(U \cap \tilde{D})=P_{p}^{-1}(B) .
$$

Donc $\tilde{\pi}(U \cap \tilde{D})$ est appliqué homéomorphiquement par $\Phi$ sur $P_{p}^{-1}(B)$, ce qui contredit l'hypothèse. Donc $R$ est vide. $\left(\tilde{D}, \tilde{\Phi}, C^{n r_{p}} \times V_{r_{p}, r_{p-1}} \times \cdots \times\right.$ $\left.V_{r_{2}, r_{1}}\right)$ est par suite un domaine localement pseudoconvexe. Grâce au théorème de Oka, $\tilde{D}$ est de Stein. Grâce au théorème de MatsushimaMorimoto, $D$ est de Stein. Ceci achève la démonstration du Théorème 2.

C'est naturel d'étendre les deux théorème au problème de Lévi pour des domaines au-dessus d'un fibré à base une variété de Stein et à fibré une variété drapeau. Mais, pour résoudre ce problème, il me semble qu'il faut savoir les isomorphismes des variétés drapeaux. Donc les résultats ne peuvent pas être donnés ici.

\section{REFERENCES}

[1] J. Brun, Sur le problème de Lévi dans certains fibrés, Manuscripta Math., 14 (1974), 217-222.

[2] J. Dieudonné, La Géométrie des Groupes Classiques, Springer-Verlag (1963).

[3] F. Docquier and H. Grauert, Levisches Problem und Rungescher Satz für Teilgebiete Steinscher Mannigfaltigkeiten, Math. Ann., 140 (1960), 94-123.

[4] H. Grauert and R. Remmert, Konvexität in der komplexen Analysis. Nicht holomorph-konvexe Holomorphiegebiete und Anwendungen auf die Abbildungestheorie, Com. Math. Helv., (1956/57), 152-183.

[5] A. Hirschowitz, Sur la géométrie analytique au-dessus des grassmannienne, C. R. A., 271 (1970), 1167-1170.

[6] _ Pseudoconvexité au-dessus d'espace plus ou moins homogènes, Invent. Math., 26 (1974), 303-322.

[7] _ Le probleme de Lévi pour les espaces homogènes, Bull. Soc. Math. France, 103 (1975), 191-201. 
[8] Y. Matsushima and A. Morimoto, Sur certains espaces fibrés holomorphes sur une variété de Stein, Bull. Soc. Math. France, 88 (1960), 137-155.

[9] K. Oka, Sur les fonctions analytiques de plusieurs variables, Iwanami Shoten, Tokyo, 1961.

[10] T. Ueda, Pseudoconvex domains over Grassmann manifolds, J. Math. Kyoto Univ., 20-2 (1980), 391-394.

Received February 16, 1982 and in revised form September 9, 1983.

UNIVERSITÉ DE NAGASAKI

NAGASAKI 852 JAPON 


\section{PACIFIC JOURNAL OF MATHEMATICS \\ EDITORS}

Donald BABBITT (Managing Editor)

University of California

Los Angeles, CA 90024

J. Dugundu

University of Southern Californa

Los Angeles, CA 90089-1113

R. FINN

Stanford University

Stanford, CA 94305

HermanN FLaSChKa

University of Arizona

Tucson, AZ 85721

\author{
C. C. MOORE \\ University of California \\ Berkeley, CA 94720 \\ ARTHur Ogus \\ University of California \\ Berkeley, CA 94720 \\ Hugo Rossi \\ University of Utah \\ Salt Lake City, UT 84112 \\ H. SAMELSON \\ Stanford University \\ Stanford, CA 94305
}

ASSOCIATE EDITORS
R. ARENS
E. F. BECKENBACH
B. H. NeUmanN
F. WOLF
K. YOSHIDA (1906-1982)

\section{SUPPORTING INSTITUTIONS}

UNIVERSITY OF ARIZONA

UNIVERSITY OF BRITISH COLUMBIA

UNIVERSITY OF OREGON

CALIFORNIA INSTITUTE OF TECHNOLOGY

UNIVERSITY OF CALIFORNIA

MONTANA STATE UNIVERSITY

UNIVERSITY OF SOUTHERN CALIFORNIA

UNIVERSITY OF NEVADA, RENO

STANFORD UNIVERSITY

UNIVERSITY OF HAWAII

NEW MEXICO STATE UNIVERSITY

UNIVERSITY OF TOKYO

UNIVERSITY OF UTAH

WASHINGTON STATE UNIVERSITY

OREGON STATE UNIVERSITY

UNIVERSITY OF WASHINGTON 


\section{Pacific Journal of Mathematics}

\section{Vol. 116, No. $1 \quad$ November, 1985}

K. Adachi, Le problème de Lévi pour les fibrés grassmanniens et les variétés

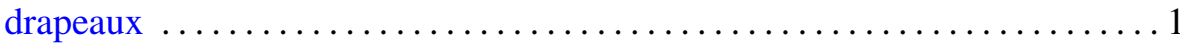

John MacLeod Ball, Remarks on the paper: "Basic calculus of variations" . . . 7 John Kelly Beem and Phillip E. Parker, Whitney stability of solvability . . . 11 Alberto Facchini, Decompositions of algebraically compact modules .......25

S. S. Khare, Finite group action and equivariant bordism $\ldots \ldots \ldots \ldots . \ldots 39$

Horst Leptin, A new kind of eigenfunction expansions on groups $\ldots \ldots \ldots . .45$

Pei-Kee Lin, Unconditional bases and fixed points of nonexpansive

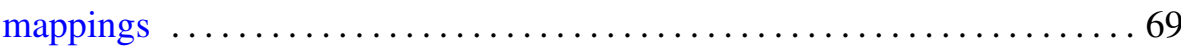

Charles Livingston, Stably irreducible surfaces in $S^{4} \ldots \ldots \ldots \ldots \ldots 77$

Kevin Mor McCrimmon, Nonassociative algebras with scalar involution . . .885

Albert Milani, Singular limits of quasilinear hyperbolic systems in a

bounded domain of $\mathbf{R}^{3}$ with applications to Maxwell's equations

Takemi Mizokami, On $M$-structures and strongly regularly stratifiable

spaces

Jesper M. Møller, On the homology of spaces of sections of complex

projective bundles

Nikolaos S. Papageorgiou, Carathéodory convex integrand operators and

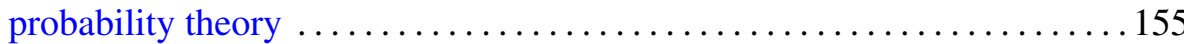

Robert John Piacenza, Transfer in generalized prestack cohomology 185

Lance W. Small and Adrian R. Wadsworth, Integrality of subrings of matrix rings ...

James Michael Wilson, On the atomic decomposition for Hardy spaces 